\title{
The Cosmological Decrease of Galactic Density and the Induced Retarded Gravity Effect on Rotation Curves
}

\author{
Asher Yahalom \\ Ariel University, Ariel 40700, Israel \\ E-mail: asya@ariel.ac.il
}

\begin{abstract}
.
Galaxies are huge physical systems having dimensions of many tens of thousands of light years. Thus any change at the galactic center will be noticed at the rim only tens of thousands of years later. Those retardation effects seems to be neglected in present day galactic modelling used to calculate rotational velocities of matter in the rims of the galaxy and surrounding gas. The significant differences between the predictions of Newtonian instantaneous action at a distance and observed velocities are usually explained by either assuming dark matter or by modifying the laws of gravity (MOND). In this paper we will show that taking general relativity seriously without neglecting retardation effects one can explain the radial velocities of galactic matter without postulating dark matter. However, this will rely on a temporal change of galactic mass. We will compare two different mechanisms of density change, one is local, that is accretion of matter from the intergalactic medium. The other is global, that is the cosmological decrease of density due to the cosmic expansion. It will be shown that local effects are much more important in this respect.
\end{abstract}

\section{Introduction}

The general theory of relativity (GR)is verified by many observations. However, at the present time, the standard Newton-Einstein gravitational theory stands at something of a crossroads. It simultaneously has much in its favor observationally, while, at the same time, it has some very disquieting challenges. The observational successes that it has achieved in both astrophysics and cosmology have to be tempered by the fact that the theory needs to appeal to two as yet unconfirmed ingredients, dark matter and dark energy, in order to achieve these successes. The dark matter problem has not only been with us since the 1920s and 1930s (when it was initially known as the missing mass problem), but it has also become more and more severe as more and more dark matter has had to be introduced on larger and larger distance scales as new data have come online. Moreover, extensive - now 40-year - underground and accelerator searches have failed to find any of it or establish its existence. The dark matter situation has become even more dire in the last few years as the Large Hadron Collider has failed to find any super symmetric particles, not only of the community's preferred form of dark matter, but also the form of it that is required in string theory, a theory that attempts to provide a quantized version of Einstein gravity.

While things may still eventually work out in favor of the standard dark matter paradigm, 
the situation is disturbing enough to warrant consideration of the possibility that the standard paradigm might at least need to be modified in some way if not outright replaced. The present proposal sets out to seek such a modification. Unlike other approaches such as Milgrom's MOND, Mannheim's Conformal Gravity or Moffat's MOG, the present approach is, in a sense, the minimalist one adhering strictly to the basic scientific principle dictated by Occam's razor. It seeks to replace dark matter by effects within standard General Relativity itself.

In 1933, Fritz Zwicky noticed that the velocities of a set of Galaxies within the Comma Cluster are much higher than those predicted by the virial calculation that assumes Newtonian theory [1]. He calculated that the amount of matter required to account for the velocities could be 400 times greater with respect to that of visible matter, which led to suggesting dark matter throughout the entire cluster. Volders, in 1959, indicated that stars in the outer rims of the nearby spiral galaxy M33 do not move "correctly" [2]. It is the result of the virial theorem coupled with Newtonian Gravity which implies that $M G / r \sim M v^{2}$, that is to say, the expected rotation curve should at some point decrease as $1 / \sqrt{r}$. This was well established during the seventies when Rubin and Ford [3, 4] demonstrated that, for a large sample of spiral galaxies, this behavior can be considered a general feature: velocities at the our rim of the galaxies do not decrease - rather, in a general case, they attain a plateau at some velocity, which is different for each galaxy.

In what follows, we will show that such effects can be deduced from GR if retardation is not neglected. The derivation of the retardation force described in previous publications $[5,6,7,34]$ is repeated here for completeness. Every natural system, such as stars and galaxies and even galactic clusters, exchanges mass with its environment. For example, the sun loses mass through solar wind and galaxies accrete gas from the intergalactic medium. This means that all natural gravitational systems have a finite retardation distance beyond which the retardation effects on gravity cannot be neglected. The question is thus quantitative: how large is the retardation distance? The change in the mass of the sun is quite small and thus the retardation distance of the solar system is quite large, allowing us to neglect retardation effects within the solar system. However, for the M33 galaxy, the velocity curve indicates that the retardation effects cannot be neglected beyond a certain distance smaller than 14,000 light years; similar analyses for other galaxies of different types have shown similar results $[8,9]$.

We notice that in the absence of temporal density changes, retardation does not effect the gravitational force. However, density is not constant for galaxies, in fact there are many processes that change the mass density in galaxies over time. Mass accretion from the inter galactic medium and internal processes such as super novae leading to super winds [9] modify the density. In addition to those local processes there is a cosmological decrease of density due to the cosmic expansion.

Here we compare two different mechanisms of density change with respect to their retardation effects. One is local, that is accretion of matter from the intergalactic medium. The other is global, that is the cosmological decrease of density due to the cosmic expansion. It will be shown that local effects are much more important in the retardation respect.

\section{General Relativity}

The general theory of relativity is based on two fundamental equations, the first being Einstein equations $[10,11,12,13]$ :

$$
G_{\mu \nu}=-\frac{8 \pi G}{c^{4}} T_{\mu \nu}
$$

$G_{\mu \nu}$ stands for the Einstein tensor, $T_{\mu \nu}$ indicates the stress-energy tensor, $G \simeq 6.67$. $10^{-11} \mathrm{~m}^{3} \mathrm{~kg}^{-1} \mathrm{~s}^{-2}$ is the universal gravitational constant and $c \simeq 310^{8} \mathrm{~ms}^{-1}$ indicates the velocity of light in the absence of matter (Greek letters are indices in the range $0-3$ ). The 
second fundamental equation that GR is based on is the geodesic equation:

$$
\frac{d^{2} x^{\alpha}}{d s^{2}}+\Gamma_{\mu \nu}^{\alpha} \frac{d x^{\mu}}{d s} \frac{d x^{\nu}}{d s}=\frac{d u^{\alpha}}{d s}+\Gamma_{\mu \nu}^{\alpha} u^{\mu} u^{\nu}=0
$$

$x^{\alpha}(s)$ are the coordinates of the particle in spacetime, $s$ is a typical parameter along the trajectory that for massive particles is chosen to be the length of the trajectory, $u^{\mu}=\frac{d x^{\mu}}{d s}$ is the $\mu$-th component of the 4-velocity of a massive particle moving along the geodesic trajectory $s$ (increment of $x$ per unit length $s$ ) and $\Gamma_{\mu \nu}^{\alpha}$ is the affine connection (Einstein summation convention is assumed). The stress-energy tensor of matter is usually taken in the form:

$$
T_{\mu \nu}=\left(p+\rho c^{2}\right) u_{\mu} u_{\nu}-p g_{\mu \nu}
$$

In the above, $p$ is the pressure and $\rho$ is the mass density. We remind the reader that lowering and raising indices is done through the metric $g_{\mu \nu}$ and inverse metric $g^{\mu \nu}$, such that $u_{\mu}=g_{\mu \nu} u^{\nu}$. The same metric serves to calculate $s$ :

$$
d s^{2}=g_{\mu \nu} d x^{\mu} d x^{\nu}
$$

and the affine connection:

$$
\Gamma_{\mu \nu}^{\alpha}=\frac{1}{2} g^{\alpha \beta}\left(g_{\beta \mu, \nu}+g_{\beta \nu, \mu}-g_{\mu \nu, \beta}\right), \quad g_{\beta \mu, \nu} \equiv \frac{\partial g_{\beta \mu}}{\partial x^{\nu}}
$$

The affine connection serves to calculate the Riemann and Ricci tensors and the curvature scalar:

$$
R_{\nu \alpha \beta}^{\mu}=\Gamma_{\nu \alpha, \beta}^{\mu}-\Gamma_{\nu \beta, \alpha}^{\mu}+\Gamma_{\nu \alpha}^{\sigma} \Gamma_{\sigma \beta}^{\mu}-\Gamma_{\nu \beta}^{\sigma} \Gamma_{\sigma \alpha}^{\mu}, \quad R_{\alpha \beta}=R_{\alpha \beta \mu}^{\mu}, \quad R=g^{\alpha \beta} R_{\alpha \beta}
$$

which, in turn, serves to calculate the Einstein tensor:

$$
G_{\alpha \beta}=R_{\alpha \beta}-\frac{1}{2} g_{\alpha \beta} R
$$

Hence, the given matter distribution determines the metric through Equation (1) and the metric determines the geodesic trajectories through Equation (2).

\section{Linear Approximation of GR}

Only in extreme cases of compact objects (black holes and neutron stars) and the primordial reality or the very early universe does one need not consider the solution of the full non-linear Einstein Equation [5]. In typical cases of astronomical interest (the galactic case included) one can use a linear approximation to those equations around the flat Lorentz metric $\eta_{\mu \nu}$ such that ${ }^{1}$ :

$$
g_{\mu \nu}=\eta_{\mu \nu}+h_{\mu \nu}, \quad \eta_{\mu \nu} \equiv \operatorname{diag}(1,-1,-1,-1), \quad\left|h_{\mu \nu}\right| \ll 1
$$

One then defines the quantity:

$$
\bar{h}_{\mu \nu} \equiv h_{\mu \nu}-\frac{1}{2} \eta_{\mu \nu} h, \quad h=\eta^{\mu \nu} h_{\mu \nu}
$$

$\bar{h}_{\mu \nu}=h_{\mu \nu}$ for non diagonal terms. For diagonal terms:

$$
\bar{h}=-h \Rightarrow h_{\mu \nu}=\bar{h}_{\mu \nu}-\frac{1}{2} \eta_{\mu \nu} \bar{h}
$$

\footnotetext{
1 Private communication with the late Professor Donald Lynden-Bell
} 
It can be shown ([10] page 75 , exercise 37 , see also $[11,12,13])$ that one can choose a gauge such that the Einstein equations are:

$$
\bar{h}_{\mu \nu, \alpha}{ }^{\alpha}=-\frac{16 \pi G}{c^{4}} T_{\mu \nu}, \quad \bar{h}_{\mu \alpha,}{ }^{\alpha}=0 .
$$

The $T_{\mu \nu}$ stress energy tensor should be calculated at the appropriate frame and thus for the rotation curve frame of reference for galaxies, matter is approximately at rest.

Equation (11) can always be integrated to take the form [14] (For reasons why the symmetry between space and time is broken, see $[15,16])$ :

$$
\begin{aligned}
& \bar{h}_{\mu \nu}(\vec{x}, t)=-\frac{4 G}{c^{4}} \int \frac{T_{\mu \nu}\left(\vec{x}^{\prime}, t-\frac{R}{c}\right)}{R} d^{3} x^{\prime}, \\
& t \equiv \frac{x^{0}}{c}, \quad \vec{x} \equiv x^{a} \quad a, b \in[1,2,3], \\
& \vec{R} \equiv \vec{x}-\vec{x}^{\prime}, \quad R=|\vec{R}| .
\end{aligned}
$$

The factor before the integral is small: $\frac{4 G}{c^{4}} \simeq 3.3 \times 10^{-44}$; hence, in the above calculation one can take $T_{\mu \nu}$, which is zero order in $h_{\alpha \beta}$. Let us now calculate the affine connection in the linear approximation:

$$
\Gamma_{\mu \nu}^{\alpha}=\frac{1}{2} \eta^{\alpha \beta}\left(h_{\beta \mu, \nu}+h_{\beta \nu, \mu}-h_{\mu \nu, \beta}\right) .
$$

The affine connection has only first order terms in $h_{\alpha \beta}$; hence, to the first order $\Gamma_{\mu \nu}^{\alpha} u^{\mu} u^{\nu}$ appearing in the geodesic, $u^{\mu} u^{\nu}$ is of the zeroth order. In the zeroth order:

$$
u^{0}=\frac{1}{\sqrt{1-\frac{v^{2}}{c^{2}}}}, u^{a}=\vec{u}=\frac{\frac{\vec{v}}{c}}{\sqrt{1-\frac{v^{2}}{c^{2}}}}, \vec{v} \equiv \frac{d \vec{x}}{d t}, \quad v=|\vec{v}| .
$$

For non relativistic velocities:

$$
u^{0} \simeq 1, \quad \vec{u} \simeq \frac{\vec{v}}{c}, \quad u^{a} \ll u^{0} \quad \text { for } \quad v \ll c .
$$

Hence, we will not be considering a post-Newtonian approximation in this paper, in which matter travels at nearly relativistic speeds, but we will be considering the retardation effects and finite propagation speed of the gravitational field. We underline that taking $\frac{v}{c}<<1$ is not the same as taking $\frac{R}{c}<<1$ (with $R$ being the typical size of a galaxy) since:

$$
\frac{R}{c}=\frac{v}{c} \frac{R}{v}
$$

and, in galaxies, $\frac{R}{v}$ is a very big number $\left(\frac{R}{v} \simeq 10^{15}\right.$ seconds); thus, $\frac{v}{c}$ can be neglected but not $\frac{R}{c}$, in which $\frac{R}{c} \simeq 10^{12}$ seconds. By inserting Equations (13) and (15) in the geodesic equation, we arrive at the approximate form:

$$
\frac{d v^{a}}{d t} \simeq-c^{2} \Gamma_{00}^{a}=-c^{2}\left(h_{0,0}^{a}-\frac{1}{2} h_{00}{ }^{a}\right)
$$

Let us now look at Equation (3). We assume $\rho c^{2} \gg p$ and, taking into account Equation (15), we arrive at $T_{00}=\rho c^{2}$, while other tensor components are significantly smaller. Thus, $\bar{h}_{00}$ is significantly larger than other components of $\bar{h}_{\mu \nu}$. One should notice that it is not possible to 
deduce from the magnitudes of quantities that such a difference exists between their derivatives. In fact, by the gauge condition in Equation (11):

$$
\bar{h}_{\alpha 0,}{ }^{0}=-\bar{h}_{\alpha a,}{ }^{a} \quad \Rightarrow \bar{h}_{00,}{ }^{0}=-\bar{h}_{0 a}{ }^{a}, \quad \bar{h}_{b 0}{ }^{0}=-\bar{h}_{b a}{ }^{a} .
$$

Thus, the zeroth derivative of $\bar{h}_{00}$ (which contains a $\frac{1}{c}$ ) is the same order as the spatial derivative of $\bar{h}_{0 a}$ and the zeroth derivative of $\bar{h}_{0 a}$ (which appears in Equation (17)) is the same order as the spatial derivative of $\bar{h}_{a b}$. However, it we can compare spatial derivatives of $\bar{h}_{00}$ and $\bar{h}_{a b}$ and conclude that the former is larger than the later. Using Equation (10) and taking into account the above consideration, we may write Equation (17) as:

$$
\frac{d v^{a}}{d t} \simeq \frac{c^{2}}{4} \bar{h}_{00,}{ }^{a} \Rightarrow \frac{d \vec{v}}{d t}=-\vec{\nabla} \phi=\vec{F}, \quad \phi \equiv \frac{c^{2}}{4} \bar{h}_{00}
$$

Thus, $\phi$ is the gravitational potential of the motion which can be calculated using Equation (12):

$$
\begin{aligned}
\phi & =\frac{c^{2}}{4} \bar{h}_{00}=-\frac{G}{c^{2}} \int \frac{T_{00}\left(\vec{x}^{\prime}, t-\frac{R}{c}\right)}{R} d^{3} x^{\prime} \\
& =-G \int \frac{\rho\left(\vec{x}^{\prime}, t-\frac{R}{c}\right)}{R} d^{3} x^{\prime}
\end{aligned}
$$

and $\vec{F}$ is the force per unit mass. If the mass density $\rho$ is static, we are in the realm of the Newtonian instantaneous action at a distance. We point out that it is unlikely that $\rho$ is static, as a galaxy will obtain mass from the intergalactic medium.

\section{Beyond the Newtonian Approximation}

The retardation time $\frac{R}{c}$ may be a few tens of thousands of years, but can be considered short in comparison to the time taken for the galactic density to change significantly. Thus, we can write a Taylor series for the density:

$$
\rho\left(\vec{x}^{\prime}, t-\frac{R}{c}\right)=\sum_{n=0}^{\infty} \frac{1}{n !} \rho^{(n)}\left(\vec{x}^{\prime}, t\right)\left(-\frac{R}{c}\right)^{n}, \quad \rho^{(n)} \equiv \frac{\partial^{n} \rho}{\partial t^{n}} .
$$

By inserting Equations (21) into Equation (20) and keeping the first three terms, we will obtain:

$$
\begin{aligned}
\phi & =-G \int \frac{\rho\left(\vec{x}^{\prime}, t\right)}{R} d^{3} x^{\prime}+\frac{G}{c} \int \rho^{(1)}\left(\vec{x}^{\prime}, t\right) d^{3} x^{\prime} \\
& -\frac{G}{2 c^{2}} \int R \rho^{(2)}\left(\vec{x}^{\prime}, t\right) d^{3} x^{\prime}
\end{aligned}
$$

The Newtonian potential is the first term, the second term has null contribution, and the third term is the lower order correction to the Newtonian theory:

$$
\phi_{r}=-\frac{G}{2 c^{2}} \int R \rho^{(2)}\left(\vec{x}^{\prime}, t\right) d^{3} x^{\prime}
$$

The total force per unit mass:

$$
\begin{aligned}
\vec{F} & =\vec{F}_{N}+\vec{F}_{r} \\
\vec{F}_{N} & =-\vec{\nabla} \phi_{N}=-G \int \frac{\rho\left(\vec{x}^{\prime}, t\right)}{R^{2}} \hat{R} d^{3} x^{\prime}, \quad \hat{R} \equiv \frac{\vec{R}}{R} \\
\vec{F}_{r} & \equiv-\vec{\nabla} \phi_{r}=\frac{G}{2 c^{2}} \int \rho^{(2)}\left(\vec{x}^{\prime}, t\right) \hat{R} d^{3} x^{\prime}
\end{aligned}
$$


While the Newtonian force $\vec{F}_{N}$ is always attractive, we notice that the retardation force $\vec{F}_{r}$ can be attractive or repulsive. The Newtonian force decreases as $\frac{1}{R^{2}}$, but the retardation force is independent of distance as long as the Taylor approximation given in Equation (21) is valid. Below a certain distance, the Newtonian force will be dominant, but for higher distances the retardation force becomes larger. Newtonian force should be neglected for distances significantly larger than the retardation distance, defined as:

$$
R \gg R_{r} \equiv c \Delta t
$$

$\Delta t$ is the typical duration in which the density $\rho$ changes; this will be defined more accurately later. On the other hand, for $R \ll R_{r}$, the retardation effect can be neglected and only Newtonian forces should be considered; this is probably the situation in the solar system. It should be stressed that the existence of $R_{r}$ does not require that velocities $v$ are high; in fact, the vast majority of galactic bodies (stars, gas) are substantially subluminal. In other words, the ratio of $\frac{v}{c} \ll 1$. Typical velocities in galaxies are $100 \mathrm{~km} / \mathrm{s}$, which makes this ratio 0.001 or smaller. However, one should consider the fact that every gravitational system, even if it is made of subluminal bodies, has a retardation distance, beyond which the retardation effect cannot be neglected. Every natural system, such as stars and galaxies and even galactic clusters, exchanges mass with its environment. For example, the sun loses mass through solar wind and galaxies accrete gas from the intergalactic medium. This means that all natural gravitational systems have a finite retardation distance. The question is thus quantitative: how large is the retardation distance? This question will be further addressed in Section 5. For large distances, $r=|\vec{x}| \rightarrow \infty$, such that $\hat{R} \simeq \frac{\vec{x}}{|\vec{x}|} \equiv \hat{r}$; thus, we obtain:

$$
\vec{F}_{r}=\frac{G}{2 c^{2}} \hat{r} \int \rho^{(2)}\left(\vec{x}^{\prime}, t\right) d^{3} x^{\prime}=\frac{G}{2 c^{2}} \hat{r} \ddot{M}, \quad \ddot{M} \equiv \frac{d^{2} M}{d t^{2}} .
$$

As the galaxy attracts intergalactic gas, its mass becomes larger and therefore $\dot{M}>0$; however, as the intergalactic gas is depleted, the rate at which the mass is accumulated must decrease and therefore $\ddot{M}<0$. Thus, in the galactic case:

$$
\vec{F}_{r}=-\frac{G}{2 c^{2}}|\ddot{M}| \hat{r}
$$

and the retardation force is attractive.

\section{Rotation Curves}

To calculate the non-asymptotic rotation curve, we note that the square of the azimuthal (orbital) velocity, $v_{\theta}$ divided by $\bar{r}$, is proportional to the derivative of the gravitational potential by $\bar{r}$, as shown in Equation (68). We use the cylindrical coordinates $\bar{r}, \theta, z$, in which $z=0$ is the galactic plane. The gravitational potential will be evaluated using Equation (22), which is composed of both the Newtonian $\phi_{N}$ and retardation $\phi_{r}$ parts, such that:

$$
\frac{v_{\theta}^{2}}{\bar{r}}=\frac{\partial \phi}{\partial \bar{r}}=\frac{\partial \phi_{N}}{\partial \bar{r}}+\frac{\partial \phi_{r}}{\partial \bar{r}},
$$

To do this, we first need a density profile describing the mass distribution in and near the galactic plane. 


\subsection{General Considerations}

We assume a density distribution of the form:

$$
\rho(\vec{x}, t)=\rho_{a}(\vec{x})+\rho_{b}(\vec{x}) g^{\prime}(t)
$$

Although the density profiles $\rho_{a}$ and $\rho_{b}$ need not have similar forms, we will assume, for simplicity, that they do. Hence, $\rho_{a}=\rho_{b}$ and, by defining $g(t)=1+g^{\prime}(t)$, we obtain a density distribution of the form:

$$
\rho(\vec{x}, t)=g(t) \rho_{a}(\vec{x})
$$

hence,

$$
M(t)=\int d^{3} x^{\prime} \rho\left(\vec{x}^{\prime}, t\right)=g(t) \int d^{3} x^{\prime} \rho_{a}\left(\vec{x}^{\prime}\right)
$$

and

$$
\ddot{M}(t)=\ddot{g}(t) \int d^{3} x^{\prime} \rho_{a}\left(\vec{x}^{\prime}\right)
$$

which leads to the result:

$$
|\ddot{g}(t)|=\frac{|\ddot{M}(t)|}{M(t)} g(t)=\frac{c^{2}}{R_{r}^{2}} g(t)
$$

here, we define:

$$
t_{r} \equiv \sqrt{\frac{M}{|\ddot{M}|}}, \quad \Delta t=t_{r} \Rightarrow R_{r} \equiv c t_{r} .
$$

Inserting Equations (30) and (33) into Equation (23) leads to the following form of the retardation potential:

$$
\phi_{r}=-\frac{G}{2 c^{2}} \ddot{g}(t) \int R \rho_{a}\left(\vec{x}^{\prime}\right) d^{3} x^{\prime}=\frac{G}{2 R_{r}^{2}} \int R \rho\left(\vec{x}^{\prime}, t\right) d^{3} x^{\prime} .
$$

Now, we introduce the dimensionless quantities:

$$
\tilde{\rho} \equiv \frac{\rho}{\rho_{c}}, \quad \tilde{x} \equiv \frac{\vec{x}}{R_{s}}
$$

in terms of a typical density $\rho_{c}$ and a typical scale $R_{s}$. From now on, we will consider all time-dependent quantities as being given at the time the galaxy is being observed. This has nothing to do with the present situation in a certain galactic system that may be millions or billions year in the future with respect to the data available to us, which of course is also the result of retardation. The dependence of the temporal variable $t$ will thus be omitted. Using Equations (36) and (31) will take the form:

$$
M=\int d^{3} x^{\prime} \rho\left(\vec{x}^{\prime}\right)=\rho_{c} R_{s}^{3} \int d^{3} \tilde{x}^{\prime} \tilde{\rho}\left(\tilde{x}^{\prime}\right)
$$

We now define the dimensionless constant:

$$
\Lambda=\int d^{3} \tilde{x}^{\prime} \tilde{\rho}\left(\tilde{x}^{\prime}\right)
$$

In terms of which:

$$
M=\Lambda \rho_{c} R_{s}^{3}
$$

Hence, $\phi_{r}$ can be written as:

$$
\phi_{r}=\frac{G M}{2 R_{r}^{2}} r \chi, \quad \chi \equiv \frac{1}{\Lambda} \int \frac{R}{r} \tilde{\rho}\left(\tilde{x}^{\prime}\right) d^{3} \tilde{x}^{\prime}
$$




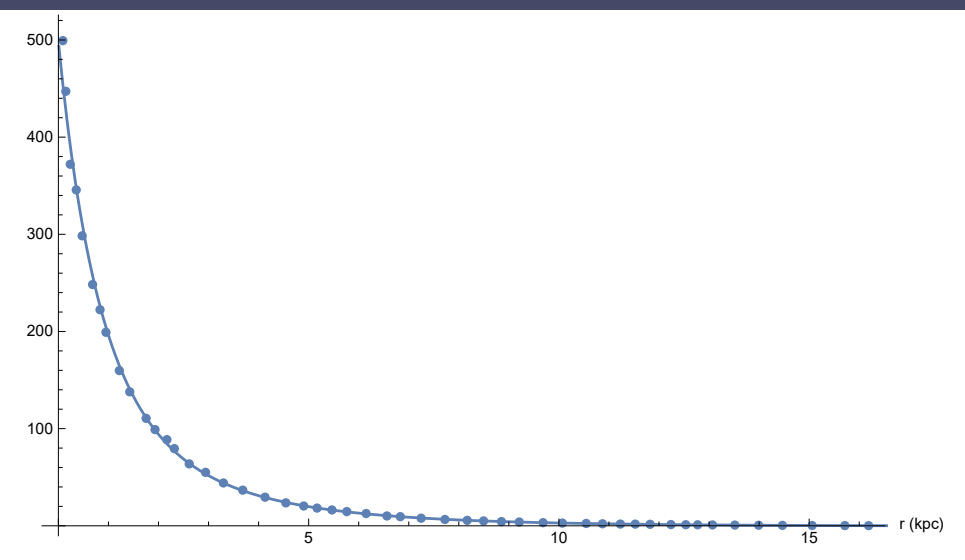

Figure 1. The M33 density radial distribution, the dots are data points [17] the solid line is a fit Equation (44).

$\chi$ is a dimensionless function that satisfies:

$$
\lim _{r \rightarrow \infty} \chi=1
$$

Similarly, one can write the Newtonian potential as:

$$
\phi_{N}=-\frac{G M}{r} \psi, \quad \psi \equiv \frac{1}{\Lambda} \int \frac{r}{R} \tilde{\rho}\left(\tilde{x}^{\prime}\right) d^{3} \tilde{x}^{\prime}
$$

$\psi$ is a dimensionless function that also satisfies:

$$
\lim _{r \rightarrow \infty} \psi=1
$$

\subsection{M33 Density Profile}

In order to calculate the retardation and Newtonian gravitational potentials and hence the rotation curve through Equation (68), we must know the density distribution in the galactic plane. This is usually done as follows: for the radial distribution, we will use the luminosity distribution and assume a proportionality constant known as the mass to light ratio. Such radial density distribution is given in Figure 1, which is based on the work of Corbelli [17] (see also Rega and Vogel [18]) and its logarithm is fitted to a polynomial of order five, as described in Equation (44):

$$
\begin{aligned}
\rho(\bar{r}) & \sim e^{k p(\bar{r})} \\
k p(\bar{r}) & =6.21207-1.05618 \bar{r}+0.137599 \bar{r}^{2}-0.0149017 \bar{r}^{3} \\
& +0.000865154 \bar{r}^{4}-0.0000209513 \bar{r}^{5}
\end{aligned}
$$

For the $z$ direction distribution orthogonal to the galactic plane, we will assume a Gaussian profile with a typical width of $\sigma=0.2 \mathrm{kpc}$. The mass distribution can be written as:

$$
\rho(\bar{r}, z)=\rho_{c} e^{k p(\bar{r})} e^{-\frac{z^{2}}{\sigma^{2}}}
$$

in which we assume cylindrical symmetry. The three-dimensional galactic mass distribution is depicted in Figure 2. 


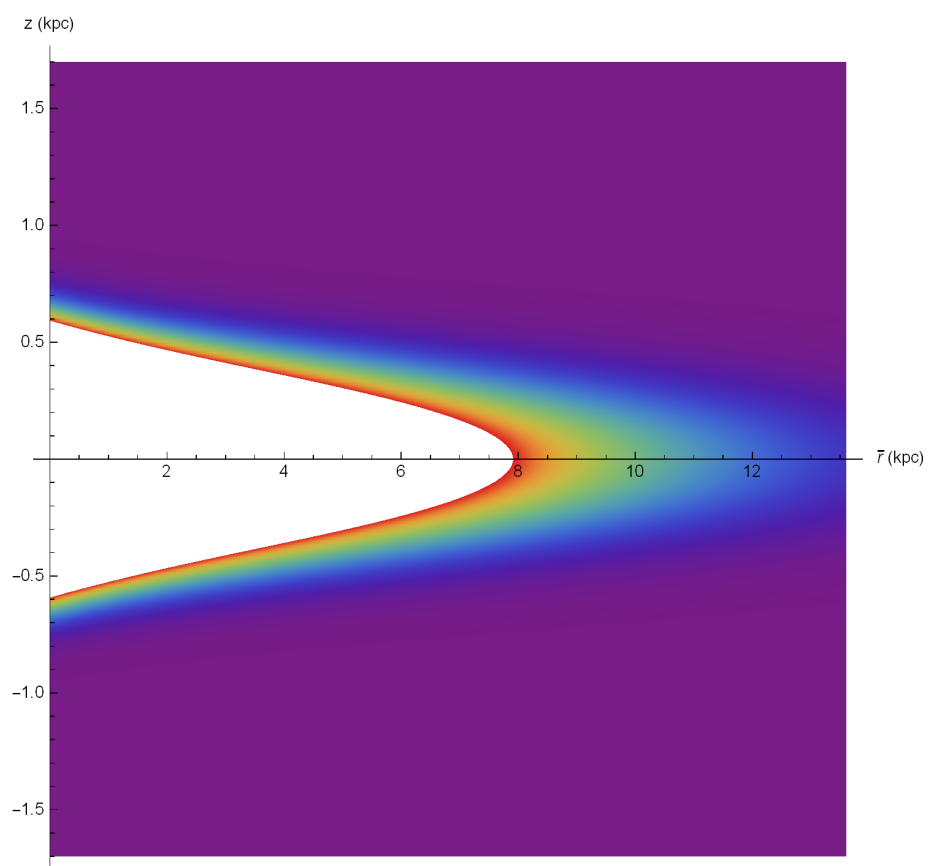

Figure 2. The M33 three dimensional mass density distribution model.

\subsection{M33 Rotation Curve}

To obtain the rotation curve we need to evaluate the functions $\psi(\bar{r})$ and $\chi(\bar{r})$, which describe the non-trivial contribution of the Newtonian and retardation potentials, respectively, see Equations (40) and (42). We will assume, for simplicity, that the galaxy is cylindrically symmetric and that rotation curves are evaluated at the galactic plane. Thus, according to Equation (42), we need to numerically evaluate the integral:

$$
\psi=\frac{1}{\Lambda} \int_{0}^{2 \pi} d \theta^{\prime} \int_{0}^{\infty} d \bar{r}^{\prime} \bar{r}^{\prime} \int_{-\infty}^{\infty} d z^{\prime} \frac{\bar{r}}{R} \tilde{\rho}, \quad \tilde{\rho}=e^{k p(\bar{r})} e^{-\frac{z^{2}}{\sigma^{2}}}
$$

in the above:

$$
\Lambda=\int_{0}^{2 \pi} d \theta^{\prime} \int_{0}^{\infty} d \bar{r}^{\prime} \bar{r}^{\prime} \int_{-\infty}^{\infty} d z^{\prime} \tilde{\rho}=0.721007
$$

and:

$$
\frac{\bar{r}}{R}=\frac{1}{\sqrt{1+\left(\frac{\bar{r}^{\prime}}{\bar{r}}\right)^{2}-2\left(\frac{\bar{r}^{\prime}}{\bar{r}}\right) \cos \left(\theta^{\prime}\right)+\left(\frac{z^{\prime}}{\bar{r}}\right)^{2}}} .
$$

The result of the numerical evaluation of $\psi(\bar{r})$ is given in Figure 3; we notice that, due to the cylindrical symmetry, it is enough to evaluate the function for the azimuthal angle $\theta=0$ as the result is similar for each azimuthal angle. As can be easily seen, the $\psi$ function of M33 converges to one for large distances, as expected from Equation (43). If we ignore the retardation potential contribution Equation (28), then Equation (42) will lead to the rotation curve depicted in Figure 4, which decreases for large distances. In the above, we assumed a galactic (baryonic) mass of $10^{10}$ solar masses $\left(2 \times 10^{40} \mathrm{~kg}\right)$ [17]. As the true galactic rotation curve does not decrease, we need to evaluate the retardation potential Equation (40) as well. For this, we evaluate the $\chi$ function as follows:

$$
\chi \equiv \frac{1}{\Lambda} \int_{0}^{2 \pi} d \theta^{\prime} \int_{0}^{\infty} d \bar{r}^{\prime} \bar{r}^{\prime} \int_{-\infty}^{\infty} d z^{\prime} \frac{R}{r} \tilde{\rho}
$$




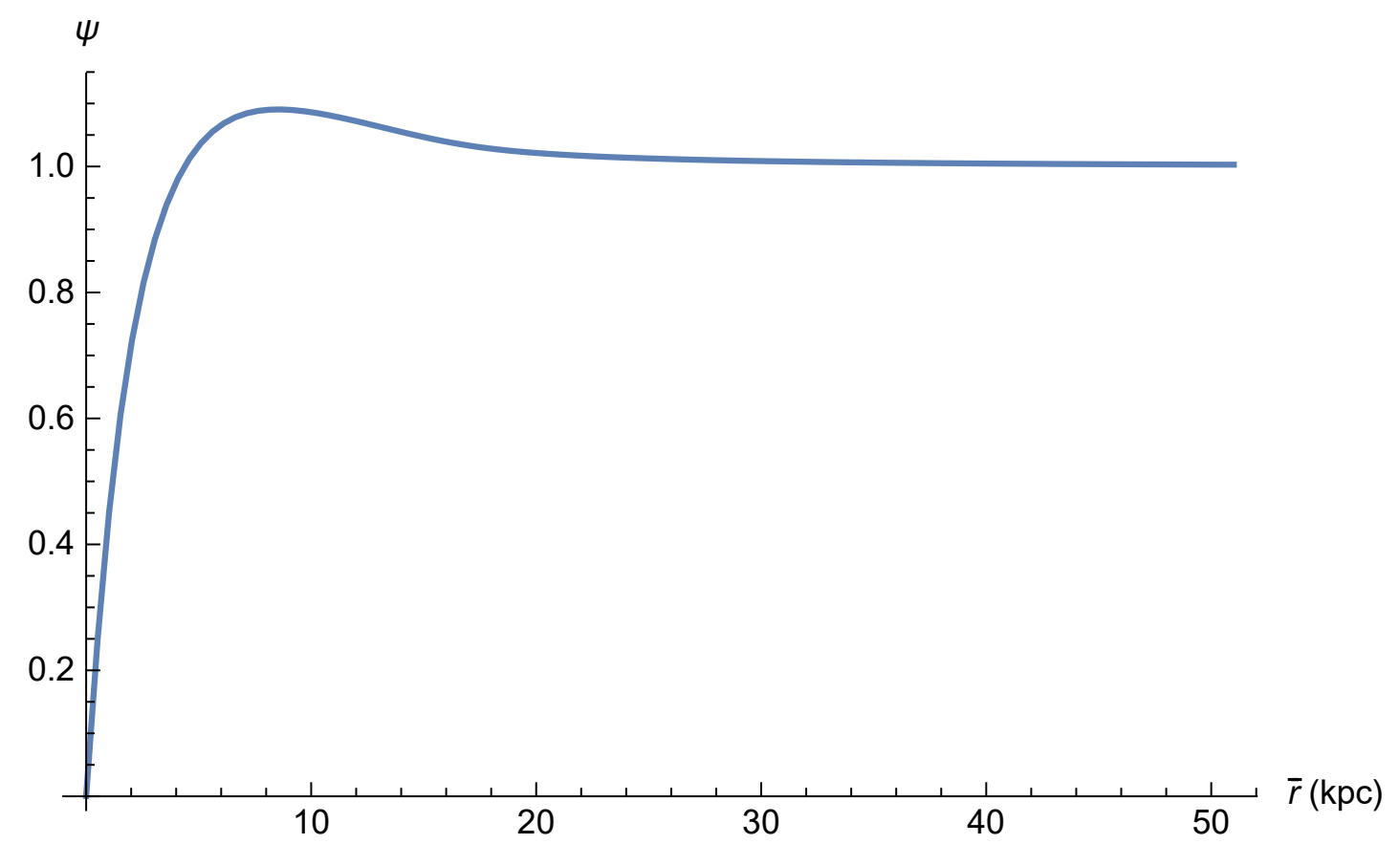

Figure 3. The $\psi$ function of M33, the function converges to one for large distances as expected from Equation (43).

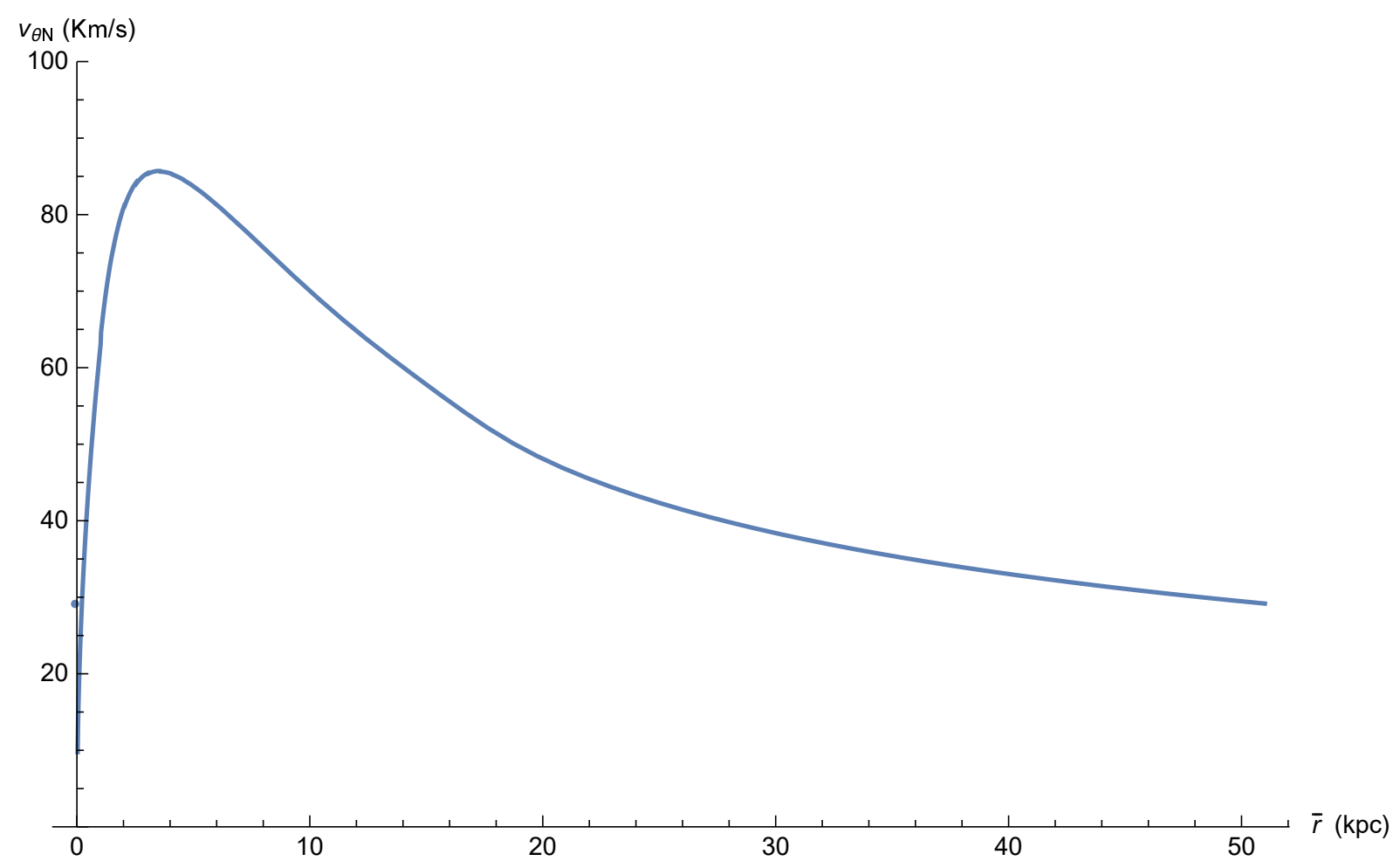

Figure 4. The Newtonian rotation curve of M33; the velocity decreases at large distances. 


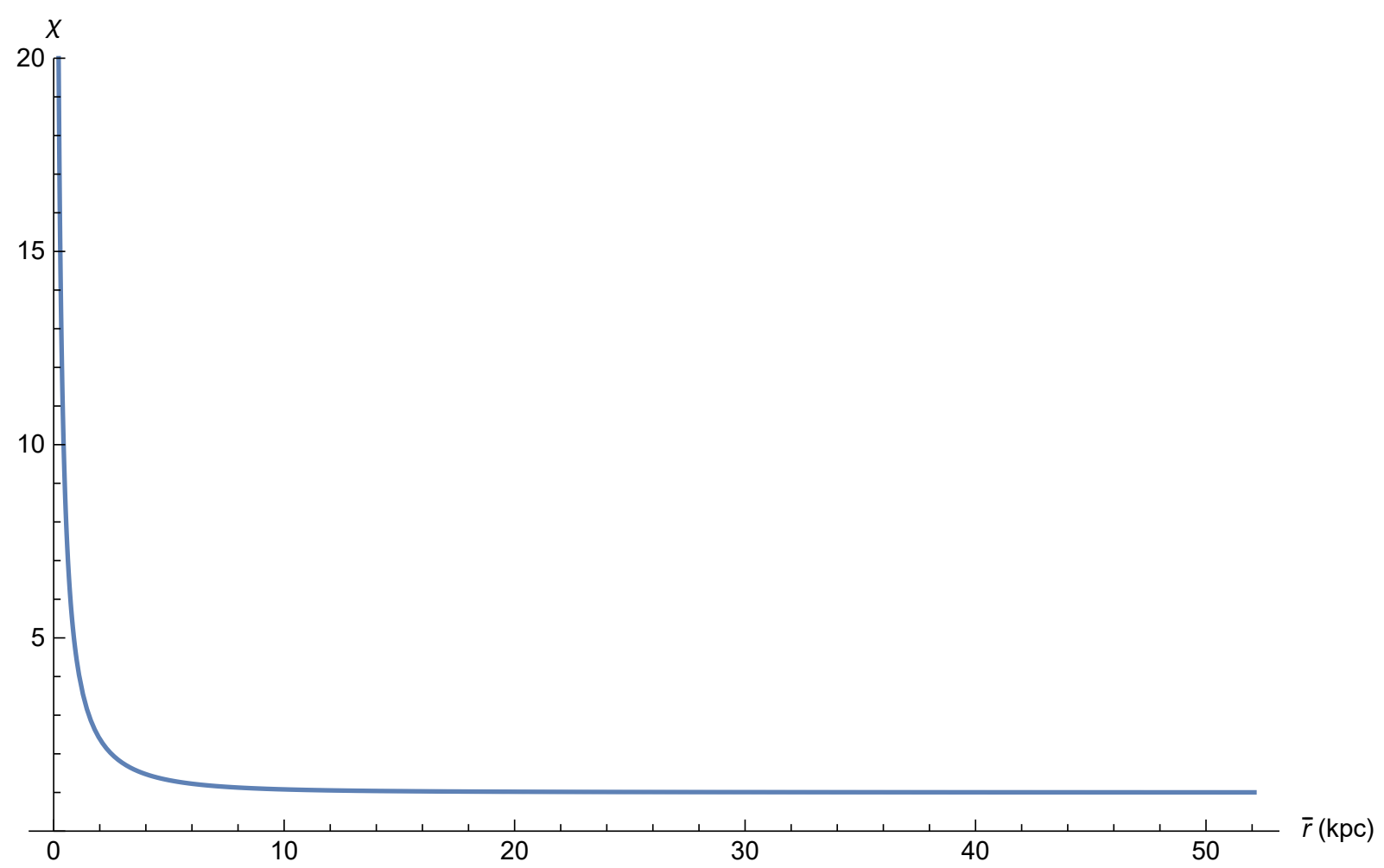

Figure 5. The $\chi$ function of M33; the function converges to one for large distances, as expected from Equation (41).

The result of the evaluation is given in Figure 5, where it is obvious that the $\chi$ function approaches unity for large distances from the galactic center. The retardation potential and thus the retardation contribution to the velocity cannot be calculated without knowledge of the retardation distance $R_{r}$. However, this can be obtained easily by fitting the observational galactic rotation curve, as demonstrated in Figure 6, which yields a best fit for $R_{r}=4.54 \mathrm{kpc}$ and a retardation time of $t_{r}=14,818.7$ years. Looking at Figure 6 , this seems quite reasonable. We deduce from the above and from equation (34) that the galactic mass second derivative is as follows:

$$
\begin{gathered}
\frac{|\ddot{M}|}{M}=\frac{1}{t_{r}^{2}} \simeq 4.6 \cdot 10^{-24} \mathrm{~s}^{-2} \\
|\ddot{M}|=\frac{M}{t_{r}^{2}} \simeq 9.12 \times 10^{16} \mathrm{~kg} / \mathrm{s}^{2}
\end{gathered}
$$

We underline that this is based only on the current estimation of the second derivative of mass; we make no claim about the past or future values of $\ddot{M}$, nor is there any claim in this section on the value of $\dot{M}$ at any time or the value of $M$ in the past or the future. It is obvious that such questions involve an understanding of the mass exchange between the galaxy and the intergalactic medium, as described in Section 6.

\section{A Dynamical Model}

The mass accumulation model described in the previous section is based on a fitting of the second derivative of the galactic mass to the galactic rotation curve. It is intuitively obvious that, as mass is accumulated in the galaxy, it must be depleted in the intergalactic medium. This 


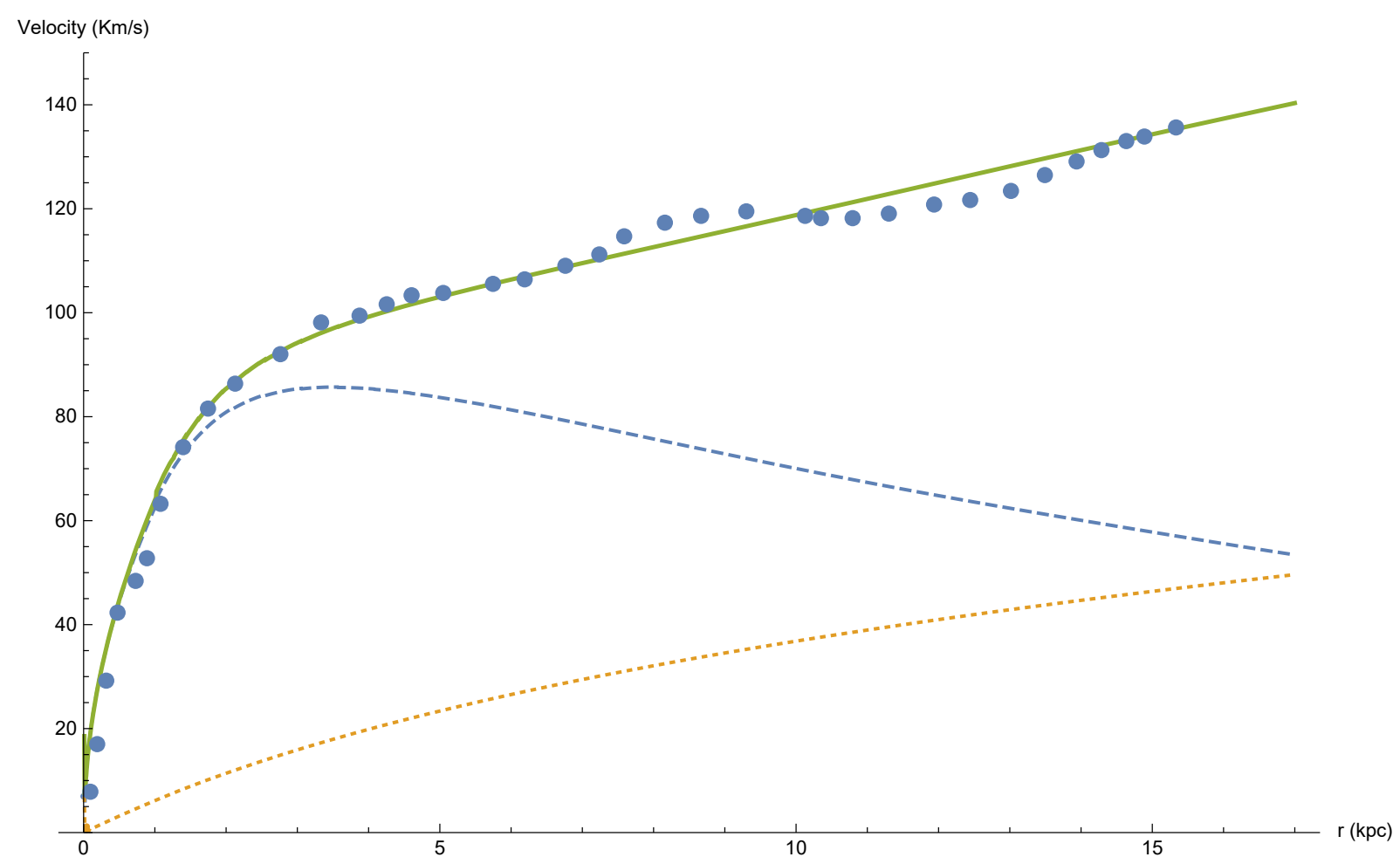

Figure 6. Rotation curve for M33. The observational points were supplied by Dr. Michal Wagman, a former PhD student at Ariel University, under my supervision, using [17]; the full line describes the complete rotation curve, which is the sum of the dotted line, describing the retardation contribution, and the dashed line, which is the Newtonian contribution.

is due to the fact that the total mass is conserved; still, it is of interest to see if this intuition is compatible with a model of gas dynamics. For simplicity, we assume that the gas is a barotropic ideal fluid and its dynamics are described by the Euler and continuity equations as follows:

$$
\begin{gathered}
\frac{\partial \rho}{\partial t}+\vec{\nabla} \cdot(\rho \vec{v})=0 \\
\frac{d \vec{v}}{d t} \equiv \frac{\partial \vec{v}}{\partial t}+(\vec{v} \cdot \vec{\nabla}) \vec{v}=-\frac{\vec{\nabla} p(\rho)}{\rho}-\vec{\nabla} \phi
\end{gathered}
$$

where the pressure $p(\rho)$ is assumed to be a given function of the density, $\frac{\partial}{\partial t}$ is a partial temporal derivative, $\vec{\nabla}$ has its standard meaning in vector analysis and $\frac{d}{d t}$ is the material temporal derivative. We have neglected viscosity terms due to the low gas density. For simplicity, we assume axial symmetry; hence, all variables are independent of $\theta$. Moreover, the mass influx coming from above and below the galaxy is much more significant compared to the influx coming from the galactic edge. This is due to the large difference in the galaxy surfaces perpendicular to the $\mathrm{z}$ axis compared to the area of its edge. The area of the surface of the galaxy which is perpendicular to the $z$ axis is:

$$
S_{z}=S_{z+}+S_{z-}=\pi r_{m}^{2}+\pi r_{m}^{2}=2 \pi r_{m}^{2}
$$

in which $S_{z}$ is the total surface area of the galaxy perpendicular to the $z$ axis, $S_{z+}$ is the upper area of the surface of the galaxy perpendicular to the $z$ axis, $S_{z-}$ is the lower area of the surface of galaxy perpendicular to the $z$ axis and $r_{m}$ is the galactic radius (see Figure 7). 


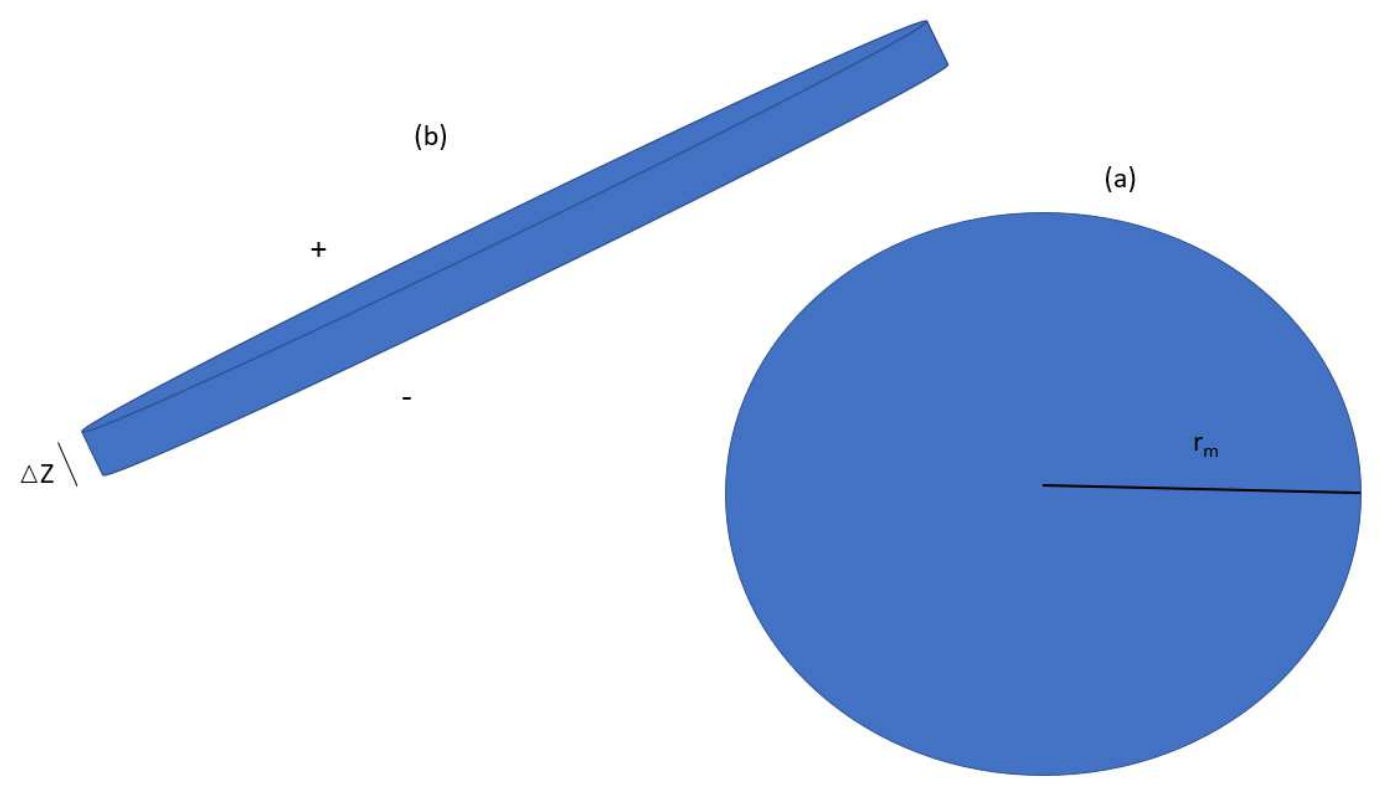

Figure 7. An idealized cylindrical galaxy from different perspectives. ( a) From above; ( b) tilted edge perspective.

The area of the surface of the galactic edge with thickness $\Delta z$ is:

$$
S_{e}=2 \pi r_{m} \Delta z
$$

Thus, the ratio of the surface area is:

$$
\frac{S_{e}}{S_{z}}=\frac{\Delta z}{r_{m}}
$$

Typical values of $\Delta z$ are about 0.4 kilo parsec and $r_{m}$ is about 17 kilo parsec (for M33), giving an area ratio of about $1 \%$. In such circumstances, the edge mass influx is less important and we can assume a velocity field of the form:

$$
\vec{v}=v_{z}(\bar{r}, z, t) \hat{z}+v_{\theta}(\bar{r}, z, t) \hat{\theta}
$$

$\hat{z}$ and $\hat{\theta}$ are unit vectors in the $z$ and $\theta$ directions, respectively. The influx is described schematically in Figure 8. In this case, the continuity Equation (52) will take the form:

$$
\frac{\partial \rho}{\partial t}+\frac{\partial\left(\rho v_{z}\right)}{\partial z}=0
$$

By defining the quantity:

$$
\gamma \equiv \rho v_{z} \Rightarrow \rho=\frac{\gamma}{v_{z}}
$$

and using the above definition, Equation (58) takes the form:

$$
\frac{\partial\left(\frac{\gamma}{v_{z}}\right)}{\partial t}+\frac{\partial \gamma}{\partial z}=0
$$




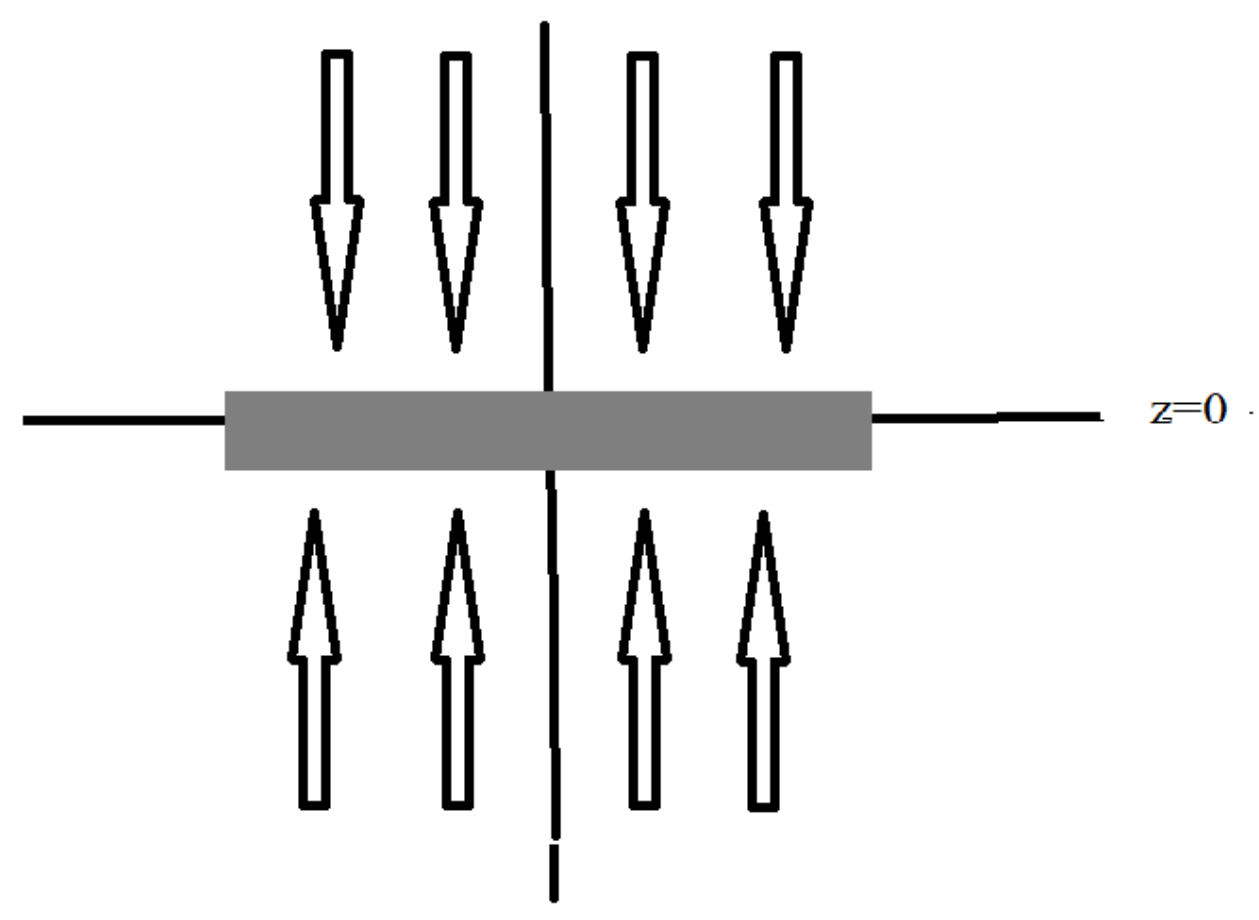

Figure 8. A schematic view of the galactic influx from the side.

Assuming, for simplicity, that $v_{z}$ is stationary and defining the auxiliary variable $t_{z}$ :

$$
t_{z} \equiv \int \frac{d z}{v_{z}}
$$

we arrive at the equations:

$$
\frac{\partial \gamma}{\partial t}+\frac{\partial \gamma}{\partial t_{z}}=0
$$

This equation can be solved easily, as follows:

$$
\gamma(\bar{r}, z, t)=f\left(t-t_{z}\right), \quad f\left(-t_{z}\right)=\gamma(\bar{r}, z, 0)=v_{z} \rho(\bar{r}, z, 0)
$$

for the function $f(x)$, which is fixed by the density initial conditions and the velocity profile.

Let us now turn our attention to the Euler Equation (53); for stationary flows, it takes the form:

$$
(\vec{v} \cdot \vec{\nabla}) \vec{v}=-\frac{\vec{\nabla} p(\rho)}{\rho}-\vec{\nabla} \phi
$$

According to Equation (57):

$$
\vec{v} \cdot \vec{\nabla}=v_{z} \frac{\partial}{\partial z}+\frac{v_{\theta}}{\bar{r}} \frac{\partial}{\partial \theta}
$$

Now, by writing Equation (64) in terms of its components, we arrive at the following equations:

$$
v_{z} \frac{\partial v_{z}}{\partial z}=-\frac{1}{\rho} \frac{\partial p}{\partial z}-\frac{\partial \phi}{\partial z}
$$




$$
-\frac{v_{\theta}^{2}}{\bar{r}}=-\frac{1}{\rho} \frac{\partial p}{\partial \bar{r}}-\frac{\partial \phi}{\partial \bar{r}}, \quad\left(\frac{\partial \hat{\theta}}{\partial \theta}=-\hat{\bar{r}}\right) .
$$

It is usually assumed that the radial pressure gradients are negligible with respect to the gravitational forces and thus we arrive at the equation:

$$
\frac{v_{\theta}^{2}}{\bar{r}} \simeq \frac{\partial \phi}{\partial \bar{r}}
$$

As for the $z$-component part of the Euler Equation (64), it can be easily written in terms of the specific enthalpy $w(\rho)=\int \frac{d P}{\rho}$ in the form:

$$
\frac{\partial}{\partial z}\left(\frac{1}{2} v_{z}^{2}+w(\rho)+\phi\right)=0 \Rightarrow \frac{1}{2} v_{z}^{2}+w(\rho)+\phi=C(r, t) .
$$

We recall that $\rho$ depends on $v_{z}$ through Equations (59) and (63):

$$
\rho(r, z, t)=\frac{\gamma}{v_{z}}=\frac{f\left(t-\int \frac{d z}{v_{z}}\right)}{v_{z}}
$$

As both the specific enthalpy and the gravitational potential are dependent on the density, Equation (69) turns into a rather complicated nonlinear integral equation for $v_{z}$. However, many galaxies are flattened structures; hence, it can thus be assumed that the pressure $z$ gradients are significant as one approaches the galactic plane. We will thus assume, for the sake of simplicity, that the pressure gradients balance the gravitational pull of the galaxy and thus $v_{z}$ is just a function of $r$, in which case the convective derivative of $v_{z}$ vanishes. The above assumption holds below and above the galactic plane, but not at the galactic plane itself. This suggests the following simple model for the velocity $v_{z}$ (see Figure 8):

$$
v_{z}=\left\{\begin{array}{cc}
-\left|v_{z}\right| & z>0 \\
\left|v_{z}\right| & z<0
\end{array}\right.
$$

in which $\left|v_{z}\right|$ is a known function of $\bar{r}$. The velocity field is discontinuous at the galactic plane due to our simplification assumptions, but, of course, need not be so in reality. We also assume, for simplicity, that the velocity field $\left|v_{z}\right|$ is constant for $\bar{r}<r_{m}$ and vanishes for $\bar{r}>r_{m}$. According to Equation (63), the time-dependent density profile is fixed by the initial density conditions. In this section, we will deal with the density profile outside the galactic plane and will leave the discussion of the density profile in and near the galactic plane to the previous section. We consider an initial density profile as follows:

$$
\begin{aligned}
\rho_{o}(\bar{r}, z, 0) & =r e(z)\left[\rho_{1}(\bar{r})+\rho_{2}(\bar{r}) e^{k|z|}\right], \\
r e(z) & = \begin{cases}1 & |z|<z_{i} \\
0 & |z| \geq z_{i}\end{cases}
\end{aligned}
$$

in which the rectangular function $r e(z)$ keeps the exponential function from diverging. The density profile is depicted in Figure 9. We assume that $\rho_{2}$ is negative and thus the density becomes dilute at distances far from the galactic plane. As $v_{z}$ is constant both above and below the galactic plane, $t_{z}=\frac{z}{v_{z}}$ up to a constant. Therefore, it is easy to deduce from Equation (63) the functional form of $f(\beta) \quad\left(\beta=-t_{z}\right)$ :

$$
f(\beta)=v_{z} r e\left(-v_{z} \beta\right)\left[\rho_{1}+\rho_{2} e^{k\left|v_{z} \beta\right|}\right]
$$




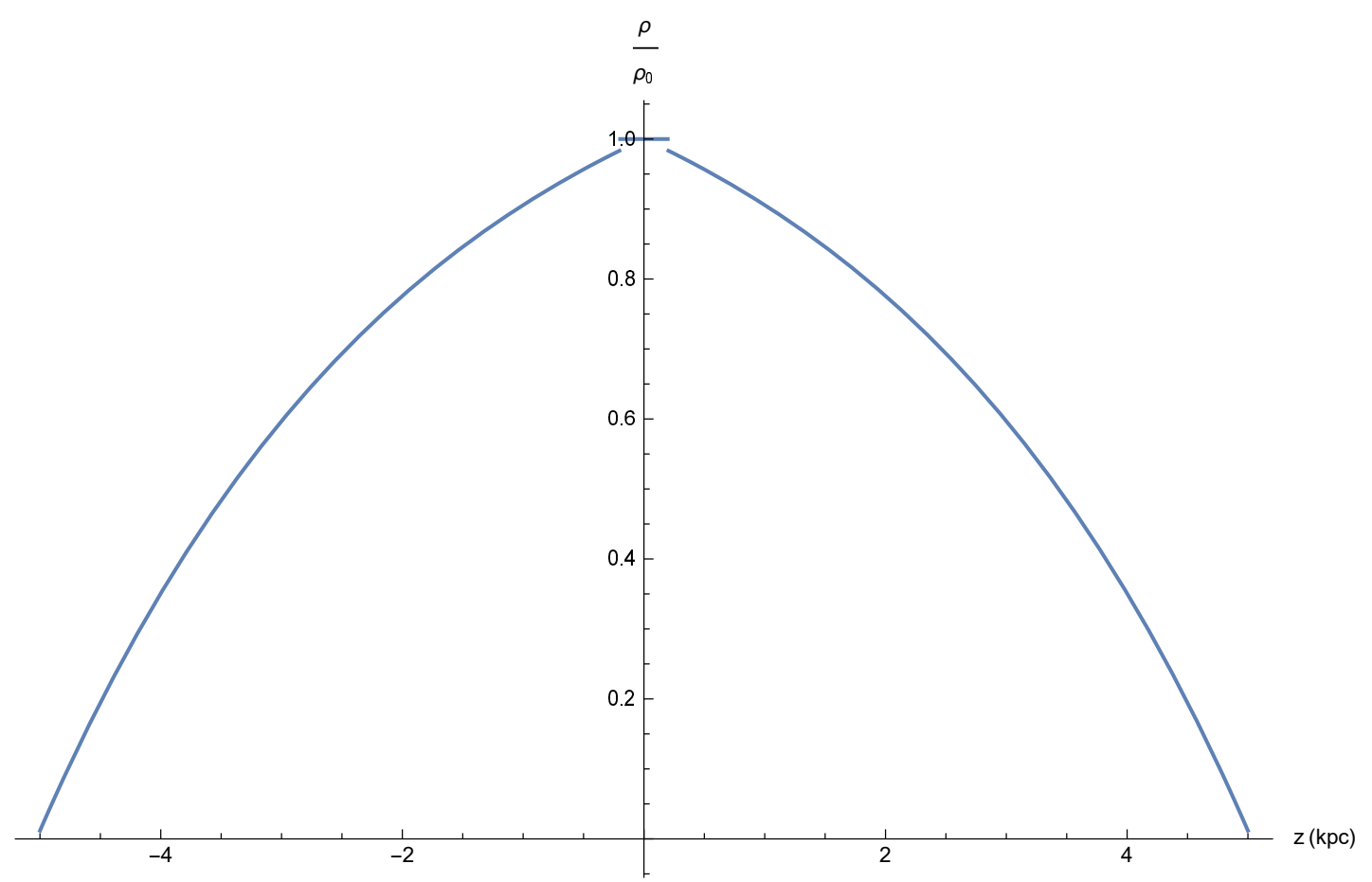

Figure 9. An initial density profile outside the galactic plane, in which $\rho_{0}=\rho_{1}+\rho_{2}, \frac{\rho_{2}}{\rho_{1}}=-0.2$ and $z_{i}=5(\mathrm{kpc})$ and $k=0.32\left(\mathrm{kpc}^{-1}\right)$.

Hence, according to Equation (70), the time-dependent density function for matter outside the galactic plane is obtained:

$$
\rho_{o}(\bar{r}, z, t)=\frac{\gamma}{v_{z}}=\operatorname{re}\left(z-v_{z} t\right)\left[\rho_{1}(\bar{r})+\rho_{2}(\bar{r}) e^{k\left|z-v_{z} t\right|}\right]
$$

The density of matter outside the galactic plane will vanish for $t>t_{m}=\frac{z_{i}}{\mid v_{z}}$; hence, we will discuss only the duration of $t<t_{m}$. Let us look at the mass contained in the cylinder defined by the galaxy (see Figure 10) and let us assume that the total mass in that cylinder is $M_{T}$. Now, the mass outside the galactic disk will be:

$$
\begin{aligned}
M_{o}(t) & =2 \pi\left[\int_{-z_{i}}^{-\frac{1}{2} \Delta z} d z \int_{0}^{r_{m}} d \bar{r} \bar{r} \rho_{o}(\bar{r}, z, t)\right. \\
& \left.+\int_{\frac{1}{2} \Delta z}^{z_{i}} d z \int_{0}^{r_{m}} d \bar{r} \bar{r} \rho_{o}(\bar{r}, z, t)\right]
\end{aligned}
$$

Hence, the mass in the galactic disk is:

$$
M(t)=M_{T}-M_{o}(t)
$$

Moreover, the galactic mass derivatives are:

$$
\dot{M}(t)=-\dot{M}_{o}(t), \quad \ddot{M}(t)=-\ddot{M}_{o}(t)
$$




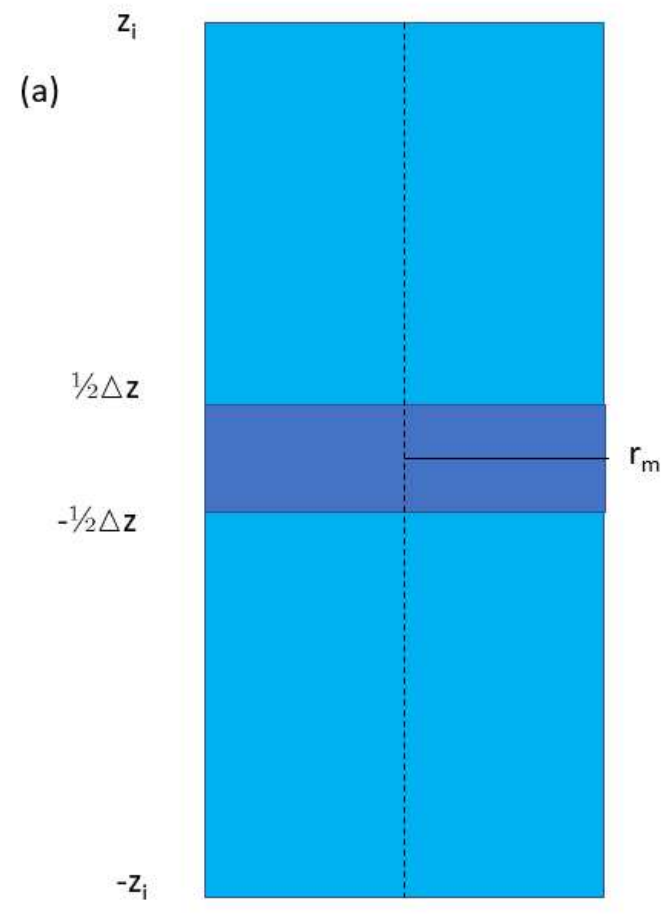

(b)

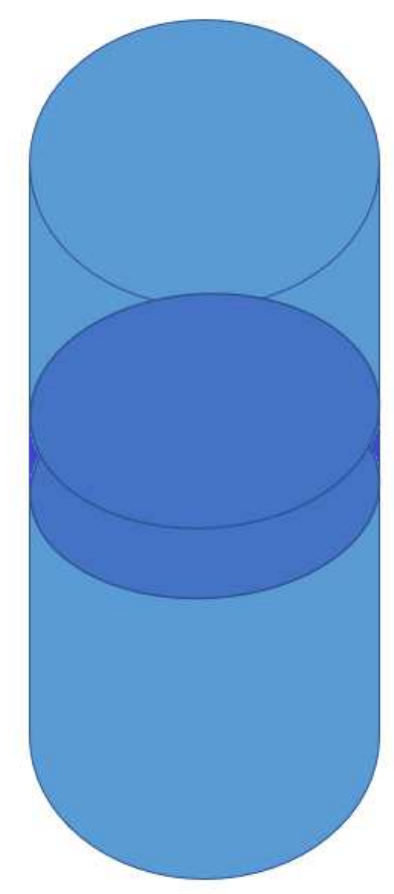

Figure 10. The mass column defined by the galaxy: ( a) side view; ( b) three-dimensional view.

By inserting Equation (74) into Equation (75), we may calculate $M_{o}(t)$ :

$$
\begin{aligned}
M_{o}(t) & =2\left[\lambda_{1}\left(z_{i}-\left|v_{z}\right| t-\frac{1}{2} \Delta z\right)\right. \\
& \left.+\frac{\lambda_{2}}{k}\left(e^{k z_{i}}-e^{k\left(\left|v_{z}\right| t+\frac{1}{2} \Delta z\right)}\right)\right]
\end{aligned}
$$

in which:

$$
\lambda_{1} \equiv 2 \pi \int_{0}^{r_{m}} d \bar{r} \bar{r} \rho_{1}(\bar{r}), \quad \lambda_{2} \equiv 2 \pi \int_{0}^{r_{m}} d \bar{r} \bar{r} \rho_{2}(\bar{r})
$$

Then, calculating the second derivative of $M_{o}(t)$ and using Equation (77) leads to the result:

$$
\ddot{M}(t)=-\ddot{M}_{o}(t)=2 k\left|v_{z}\right|^{2} e^{\frac{1}{2} \Delta z k} \lambda_{2} e^{k\left|v_{z}\right| t} .
$$

We then denote:

$$
\alpha \equiv k\left|v_{z}\right|, \quad \tau \equiv \frac{1}{\alpha}, \quad \ddot{M}(0)=2 k\left|v_{z}\right|^{2} e^{\frac{1}{2} \Delta z k} \lambda_{2} .
$$

Thus:

$$
\ddot{M}(t)=\ddot{M}(0) e^{\frac{t}{\tau}} .
$$

It should be stressed that the current approach does not require that the velocity $\left|v_{z}\right|$ is high; in fact, the vast majority of galactic bodies (stars, gas) are substantially subluminal - in other words, the ratio of $\frac{\left|v_{z}\right|}{c} \ll 1$. The typical velocities in galaxies are $100 \mathrm{~km} / \mathrm{s}$, which makes this ratio 0.001 or smaller. However, we stress again the fact that every gravitational 
system, even if it is made of subluminal bodies, has a finite retardation distance, beyond which the retardation effect cannot be neglected. As demonstrated above, a galaxy exchanges mass with its environment. This means that a galaxy has a finite retardation distance. The question is thus quantitative: how large is the retardation distance? For the M33 galaxy, the velocity curve indicates that retardation effects cannot be neglected beyond a certain distance, which is calculated in Section 5 to be roughly 14,000 light years; similar analyses for other galaxies of different types have shown similar results [8]. We demonstrate here, using a detailed model, that this does not require a high velocity of gas or stars, in or out of the galaxy, and is perfectly consistent with the current observational knowledge of galactic and extragalactic material content and dynamics. Equation (81) means that we must have $\lambda_{2}<0$ according to Equation (27) in order to assure an attractive force. Next, we calculate $\dot{M}(t)$; using Equations (77) and (78), we obtain:

$$
\dot{M}(t)=-\dot{M}_{o}(t)=2\left|v_{z}\right| \lambda_{1}+2\left|v_{z}\right| e^{\frac{1}{2} \Delta z k} \lambda_{2} e^{k\left|v_{z}\right| t} .
$$

Hence:

$$
\dot{M}(0)=2\left|v_{z}\right| \lambda_{1}+2\left|v_{z}\right| e^{\frac{1}{2} \Delta z k} \lambda_{2}=2\left|v_{z}\right| \lambda_{1}+\frac{\ddot{M}(0)}{\alpha}
$$

Thus, $\lambda_{1}$ is:

$$
\lambda_{1}=\frac{1}{2\left|v_{z}\right|}\left[\dot{M}(0)-\frac{\ddot{M}(0)}{\alpha}\right]
$$

Inserting Equations (85) and (81) into Equation (83) leads to:

$$
\dot{M}(t)=\dot{M}(0)+\tau \ddot{M}(0)\left(e^{\frac{t}{\tau}}-1\right)=\dot{M}(0)-\tau|\ddot{M}(0)|\left(e^{\frac{t}{\tau}}-1\right) .
$$

Finally, by combining Equations (76), (78), (81) and (85) and noticing that:

$$
M(0)=M_{T}-\frac{z_{i}-\frac{1}{2} \Delta z}{\left|v_{z}\right|}\left(\dot{M}(0)-\frac{\ddot{M}(0)}{\alpha}\right)-\frac{\ddot{M}(0)}{\alpha^{2}}\left(e^{k\left(z_{i}-\frac{1}{2} \Delta z\right)}-1\right)
$$

we arrive at:

$$
M(t)=M(0)+(\dot{M}(0)-\tau \ddot{M}(0)) t+\tau^{2} \ddot{M}(0)\left(e^{\frac{t}{\tau}}-1\right), \quad \tau>0 .
$$

\section{Cosmic Expansion and The Decrease of Density}

In section 3 we assumed the background metric is Lorentzian, however, this is not so because the universe experiences a Hubble (accelerated) expansion. This expansion can be explained in terms of the Friedman-Lemaitre-Robertson-Walker metric. We choose following Narlikar [10], the non-perturbed metric to be:

$$
g_{\mu \nu}^{(0)}=\operatorname{diag}\left(1,-\frac{S^{2}(t)}{1-k r^{\prime 2}},-S^{2}(t) r^{\prime 2},-S^{2}(t) r^{\prime 2} \sin ^{2} \theta\right)
$$

for the coordinates $x^{\nu}=\left(c t, r^{\prime}, \theta, \phi\right)$ and $S(t)$ is a scaling factor. In which case the complete metric will be:

$$
g_{\mu \nu}=g_{\mu \nu}^{(0)}+h_{\mu \nu} .
$$

Now, mass conservation dictates that:

$$
\rho(\vec{x}, t) S^{3}(t)=\rho\left(\vec{x}, t_{0}\right) S^{3}\left(t_{0}\right)
$$


Or:

$$
\rho(\vec{x}, t)=\rho\left(\vec{x}, t_{0}\right) \frac{S^{3}\left(t_{0}\right)}{S^{3}(t)} .
$$

Taking the first temporal partial derivative we arrive at:

$$
\frac{\partial \rho}{\partial t}=-3 \rho H \quad H \equiv \frac{\dot{S}}{S},
$$

in which $H$ is the Hubble "constant". The second partial derivative would be:

$$
\frac{\partial^{2} \rho}{\partial t^{2}}=3 \rho\left[4 H^{2}-\frac{\ddot{S}}{S}\right]
$$

Hence:

$$
\frac{\ddot{M}}{M}=3\left[4 H^{2}-\frac{\ddot{S}}{S}\right] .
$$

We remark that for an accelerating universe $\ddot{S}>0$. The retardation distance and duration are thus dependent on universe scaling factor and hence on the Hubble constant and universe acceleration. The $\Lambda$ CDM model seems to give a reasonable estimation of the scale factor according to the analysis of cosmic microwave background and distant super novae data [35], and we will use it here without admitting to the ontological existence of "dark matter" or "dark energy". Assuming a flat universe $(k=0)$ and a simple dark energy model $(w=1)$ and neglecting a minuscule amount of radiation density we arrive at:

$$
H=\frac{\dot{S}}{S}=H_{0} \sqrt{\Omega_{m} S^{-3}+\Omega_{\Lambda}} .
$$

In which $H_{0}=67.74 \pm 0.46 \mathrm{~km} \mathrm{~s}^{-1} M p c^{-1}, \Omega_{m}=0.3089 \pm 0.0062$ and $\Omega_{\Lambda}=0.6911 \pm 0.0062$. This equation is solved as follows:

$$
S(t)=\left(\frac{\Omega_{m}}{\Omega_{\Lambda}}\right)^{\frac{1}{3}} \sinh ^{\frac{2}{3}}\left(\frac{t}{t_{\Lambda}}\right)
$$

where $t_{\Lambda} \equiv 2 /\left(3 H_{0} \sqrt{\Omega_{\Lambda}}\right) \simeq 11.6$ Gyears, thus up to about $10 \%$ error in the current age of the universe:

$$
S(t) \simeq(0.5)^{\frac{2}{3}}\left(\frac{\Omega_{m}}{\Omega_{\Lambda}}\right)^{\frac{1}{3}} e^{\frac{2}{3} \frac{t}{t_{\Lambda}}}
$$

Thus according to equation (95) we have:

$$
\frac{\ddot{M}}{M} \simeq \frac{4}{t_{\Lambda}^{2}} \simeq 510^{-35}
$$

This should be compared to equation (50) showing that unlike the attractive depletion contribution the cosmological contribution is repulsive and is about eleven orders of magnitude smaller hence negligible. 


\section{Conclusions}

The need to satisfy the Lorentz symmetry group prevents the weak field approximation of GR from allowing action at distance potentials and thus only retarded solutions are allowed. Retardation is manifested more strongly when large distances and large second derivatives are involved. It should be stressed that the current approach does not require that velocities, $v$ are high; in fact, the vast majority of galactic bodies (stars, gas) are substantially subluminal -in other words, the ratio of $\frac{v}{c} \ll 1$. The typical velocities in galaxies are $100 \frac{\mathrm{km}}{\mathrm{s}}$ (see Figure 6 ), which makes this ratio 0.001 or smaller. However, one should consider the fact that every gravitational system, even if it is made of subluminal bodies, has a retardation distance, beyond which the retardation effect cannot be neglected. Every natural system, such as a star or a galaxy and even a galactic cluster, exchanges mass with its environment. For example, the sun loses mass through solar wind and galaxies accrete gas from the intergalactic medium. This means that all natural gravitational systems have a finite retardation distance. The question is thus quantitative: how large is the retardation distance? The change in the mass of the sun is quite small and thus the retardation distance of the solar system is huge, allowing us to neglect retardation effects within the solar system. However, for the M33 galaxy, the velocity curve indicates that the retardation effects cannot be neglected beyond a certain distance, which was calculated in Section 5 to be roughly $R_{r}=4.54 \mathrm{kpc}$; similar analyses for other galaxies of different types have shown similar results $[8,9]$. We demonstrated, using a detailed model, in Section 6, that this does not require a high velocity of gas or stars in or out of the galaxy and is perfectly consistent with the current observational knowledge of galactic and extragalactic material content and dynamics.

We point out that, if the mass outside the galaxy is still abundant (or totally consumed), $\ddot{M} \simeq 0$ and retardation force should vanish. This was reported [19] for the galaxy NGC1052-DF2.

We note that the same terms in the gravitation equation that are responsible for the gravitational radiation recently discovered are also responsible for the rotation curves of galaxies. The expansion given in Equation (22), being a Taylor series expansion up to the second order, is only valid for limited radii:

$$
R<c T_{\max } \equiv R_{\max }
$$

This means that current expansion is related to the near field case; this is acceptable since the extension of the rotation curve in galaxies is the same order of magnitude as the size of the galaxy itself. An opposite case in which the size of the object is much smaller than the distance to the observer will result in a different approximation to Equation (12), leading to the famous quadruple equation of gravitational radiation, as predicted by Einstein [22] and verified indirectly in 1993 by Russell A. Hulse and Joseph H. Taylor, Jr., for which they received the Nobel Prize in Physics. The discovery and observation of the Hulse-Taylor binary pulsar offered the first indirect evidence of the existence of gravitational waves [23]. On 11 February 2016, the LIGO and Virgo Scientific Collaboration announced that they had made the first direct observation of gravitational waves. The observation was made five months earlier, on 14 September 2015, using Advanced LIGO detectors. The gravitational waves originated from the merging of a binary black hole system [24]. Thus, the current paper involves a near-field application of gravitational radiation while previous works discuss far- field results.

We regret that direct measurement of the second temporal derivative of the galactic mass is not available. What is available is the remarkable fit between the retardation model and the galactic rotation curve, as can be seen in Figure 6, which constitutes indirect evidence of the galactic mass second derivative. The reader is reminded that competing theories like dark matter do not supply any observational evidence either. Despite the work of thousands of people and the investment of billions of dollars, there is still no evidence of dark matter. Occam's razor dictates that when two theories compete, the one that makes less assumptions has the upper hand. In the case of retardation theory, only baryonic matter and a large second temporal derivative of mass are assumed. 
Problems that inflict dark matter theory, such as the cuspy halo problem (also known as the core-cusp problem) refers to a discrepancy between the inferred dark matter density profiles of low-mass galaxies and the density profiles predicted by cosmological N-body simulations. Nearly all simulations form dark matter halos, which have "cuspy" dark matter distributions, with density increasing steeply at small radii, while the rotation curves of most observed dwarf galaxies suggest that they have flat central dark matter density profiles ("cores"). This problem does not occur in the retardation model which denies the existence of dark matter. One cannot discuss flat or sharp profiles of dark matter if dark matter does not exist. The inherent problems with dark matter's dynamics further strengthen the claim of this work that dark matter does not exist and the rotation curve characteristics attributed to dark matter should be attributed to retardation.

How can one reach an erroneous conclusion regarding the existence of "dark matter"? By ignoring retardation effects and assuming that radial velocities are a result of some mysterious substance. We obtain for a spherically symmetric distribution [25]:

$$
-\frac{v_{c}^{2}}{r} \hat{r}=\vec{F}_{d}=-\frac{G M_{d}(r)}{r^{2}} \hat{r}
$$

where $v_{c}$ is the speed of a particle of unchanging radius $r$ and $M_{d}(r)$ is the dark matter within the radius $r$. Comparing Equations (101) and (27), we deduce that the "dark matter" mass can be calculated as follows:

$$
M_{d}(r)=\frac{r^{2}|\ddot{M}|}{2 c^{2}}
$$

Then, since:

$$
M_{d}(r)=4 \pi \int_{0}^{r} r^{\prime 2} \rho_{d}\left(r^{\prime}\right) d r^{\prime}, \quad \frac{d M_{d}(r)}{d r}=4 \pi r^{2} \rho_{d}(r)
$$

it follows that:

$$
\rho_{d}(r)=\frac{|\ddot{M}|}{4 \pi c^{2} r}
$$

This is consistent with the observational data of [26] who concluded that the "dark matter" density decreases as $r^{-1.3}$ for M33.

An additional method to explaining galactic rotation curves is to postulate that either the laws of dynamics or the laws of gravitation (GR) should be changed. This is the case in an approach championed by Milgrom (modifying the laws of gravity (MOND) - modified Newtonian dynamics) [27]. In this approach, the classical law of gravity is modified:

$$
\vec{F}_{M}=-\frac{G M}{\mu\left(\frac{a}{a_{0}}\right) r^{2}} \hat{r}
$$

In the above $\mu$, is the interpolation function that should be one for $a_{0} \ll a$. Let us assume:

$$
\mu(x)=\frac{x}{\sqrt{1+x^{2}}} \Rightarrow \mu\left(\frac{a}{a_{0}}\right)=\frac{1}{\sqrt{1+\left(\frac{a_{0}}{a}\right)^{2}}}
$$

if $a_{0} \gg a, \mu \simeq \frac{a}{a_{0}}$. A particle revolving in a unchanging radius will have a centrifugal acceleration of $a=\frac{v^{2}}{r}$ and thus:

$$
\vec{F}_{M}=-\frac{G M a_{0}}{v^{2} r} \hat{r}
$$

For the constant $v$ at a far away distance, this expression is similar to the retardation force and thus:

$$
|\ddot{M}|=\frac{2 M a_{0} c^{2}}{v^{2} r} .
$$


Milgrom found $a_{0}=1.2 \times 10^{-10} \mathrm{~ms}^{-2}$ to be most fitting to the data. The velocity at $15.33 \mathrm{kpc}$ from the center of the galaxy is $135,640 \mathrm{~ms}^{-1}$. We thus obtain $|\ddot{M}| \simeq 4.94 \times 10^{16} \mathrm{kgs}^{-2}$ and a retardation time of:

$$
t_{r}=\sqrt{\frac{M}{|\ddot{M}|}} \simeq 6.3510^{11} \mathrm{~s}
$$

This amounts to a typical accumulation acceleration time scale of $t_{r} \simeq 20,129$ years and a retardation distance of:

$$
R_{r}=c t_{r} \simeq 20,129 \text { light years, }
$$

which seems reasonable according to our fitting estimates. Hence, despite the fact that modified Newtonian dynamics theory is phenomenological and is in contradiction to general relativity it can still serve as tool for estimating retardation theory quantities.

We have thus shown that "dark matter" and "MOND" effects can be explained in the framework of standard GR as effects due to retardation, without assuming any exotic matter or modifications of the theory of gravity.

To conclude this section, we would like to mention the remarkable theory of conformal gravity put forward by Mannheim [29, 30]. The current retardation approach leads to a Newtonian potential plus a linear potential. Such potential types can also be derived from the completely different theoretical considerations of conformal gravity. Indeed, on purely phenomenological grounds, such fits have essentially already been published in the literature. While those fits looked very good, they had to treat the coefficient of the linear potential as a variable that changed from galaxy to galaxy; this element is not in line with conformal gravity in which the coefficient of the linear potential is a new universal constant of nature. This can be explained much more easily in the framework of retardation theory, in which a variable $\ddot{M} / M$ seems reasonable depending on the dynamical conditions of various galaxies. Indeed, $\ddot{M} / M$ has a theoretical basis, while a pure phenomenological approach does not. As far as we understand the work of Mannheim [28, 29, 30], it is related to conformal gravity, which is different from GR, and thus has to justify other results of GR (Big Bang Cosmology, etc.). We also underline that retardation theory does not contradict conformal gravity and, in reality, both effects may exist, although Occam's razor forbids us to add new universal constants if the existing ones suffice to explain observations.

Retardation theory's approach is minimalistic (in the sense that it satisfies the Occam's razor rule), does not affect observations that are beyond the near-field regime and, therefore, does not clash with GR theory and its observations (nor with Newtonian theory, as the retardation effect is negligible for small distances). We underline that a perfect fit to the rotation curve is achieved with a single parameter and we do not adjust the mass to light ratio in order to improve our fit as other authors do.

In the current paper we study the effect of the Hubble expansion of space-time on the decrease of density and hence on retardation. We show that unlike the effect of depletion this phenomenon causes repulsive gravity and is negligible with respect to the former retardation effect.

Retardation effects in electromagnetic theory were discussed in [31, 32, 33].

Finally the paper does not discuss dark matter in a cosmological context (but it does take into account cosmological effects in a galaxy) or in a gravitational lensing scenario; this is left for future works. Another important application is the study of galaxy clusters. This issue led Zwicky [1] to suggest dark matter in the first place.

\section{Acknowledgement}

The author wishes to thank his former student, Michal Wagman, for supplying the data points for the rotation curve of the M33 galaxy. This work is a result of more than twenty 
five years of thinking (discontinuously) on the dark matter problem, which was first suggested to me by the late Jacob Bekenstein during my stay at the Hebrew University of Jerusalem. The current retardation solution arose from my discussions with the late Donald Lynden-Bell of Cambridge University, and the late Miron Tuval. Other people with which I discussed this work and offered important feedback are Lawrence Horwitz of Tel-Aviv University and Marcelo Shiffer of Ariel University. Recently, this work benefitted from discussions with James Peebles, Neta Bachall and Sam Cohen, all from Princeton University. Special thanks are due to Jiri Bicak for inviting me to present the theory at Charles University in Prague. I would like to thank Philip Mannheim and James Obrien for our discussions during the recent IARD meetings and for supplying some relevant data. I have benefited from discussions with a very long list of distinguished scientists and ask their forgiveness for not mentioning them all.

[1] Zwicky, F. On a New Cluster of Nebulae in Pisces. Proc. Natl. Acad. Sci. USA 1937, 23, 251-256.

[2] Volders, L.M.J.S. Neutral Hydrogen in M33 and M101. Bull. Astr. Inst. Netherl. 1959, 14, 323.

[3] Rubin, V.C.; Ford, W.K., Jr. Rotation of the Andromeda Nebula from a Spectroscopic Survey of Emission Regions. Astrophys. J. 1970, 159, 379.

[4] Rubin, V.C.; Ford, W.K., Jr.; Thonnard, N. Rotational Properties of 21 Sc Galaxies with a Large Range of Luminosities and Radii from NGC $4605(\mathrm{R}=4 \mathrm{kpc})$ to UGC $2885(\mathrm{R}=122 \mathrm{kpc})$. Astrophys. J. 1980, 238, 471.

[5] Yahalom, A. The effect of Retardation on Galactic Rotation Curves. J. Phys.: Conf. Ser. 1239 (2019) 012006.

[6] Yahalom, A. Retardation Effects in Electromagnetism and Gravitation. In Proceedings of the Material Technologies and Modeling the Tenth International Conference, Ariel University, Ariel, Israel, 20-24 August 2018. (arXiv:1507.02897v2)

[7] Yahalom, A. Dark Matter: Reality or a Relativistic Illusion? In Proceedings of Eighteenth Israeli-Russian Bi-National Workshop 2019, The Optimization of Composition, Structure and Properties of Metals, Oxides, Composites, Nano and Amorphous Materials, Ein Bokek, Israel, 17-22 February 2019.

[8] Wagman, M. Retardation Theory in Galaxies. Ph.D. Thesis, Senate of Ariel University, Ariel, Israel, 23 September 2019.

[9] Wagman, M.; Horwitz, L.P.; Yahalom, A. Retardation Theory and galactic Rotation Curves. submitted to Astrophys. J.

[10] Narlikar, J.V. Introduction to Cosmology, 2nd ed.; Cambridge University Press: Cambridge, UK, 1993.

[11] Eddington, A.S. The Mathematical Theory of Relativity; Cambridge University Press: Cambridge, UK, 1923.

[12] Weinberg, S. Gravitation and Cosmology: Principles and Applications of the General Theory of Relativity; John Wiley \& Sons, Inc.: Hoboken, NJ, USA, 1972.

[13] Misner, C.W.; Thorne, K.S.; Wheeler, J.A. Gravitation; W.H. Freeman \& Company: New York, NY, USA, 1973.

[14] Jackson, J.D. Classical Electrodynamics, 3rd ed.; Wiley: New York, NY, USA, 1999.

[15] Asher, Y. The Geometrical Meaning of Time. Found. Phys. 2008, 38, 489-497.

[16] Yahalom, A. The Gravitational Origin of the Distinction between Space and Time. Int. J. Mod. Phys. D 2009, 18, 2155-2158.

[17] Corbelli, E. Monthly Notices of the Royal Astronomical Society 2003, 342, 199-207, doi:10.1046/j.13658711.2003.06531.x.

[18] Rega, M.W.; Vogel, S.N. Astrophysical Journal 1994, 434, 536.

[19] van Dokkum, P.; Danieli, S.; Cohen, Y.; Merritt, A.; Romanowsky, A.J.; Abraham, R.; Brodie, J.; Conroy, C.; Lokhorst, D.; Mowla, L.; et al. A galaxy lacking dark matter. Nature 2018, 555, 629-632, doi:10.1038/nature25767.

[20] Fodera-Serio, G.; Indorato, L.; Nastasi, P. Hodierna's Observations of Nebulae and his Cosmology. J. Hist. Astron. 1985, 16, 1-36, doi:10.1177/002182868501600101.

[21] Van den Bergh, S. The Galaxies of the Local Group; Cambridge Astrophysics Series 35; Cambridge University Press: Cambridge, UK, 2000; p. 72. ISBN 978-0-521-65181-3.

[22] Einstein, A. Näherungsweise Integration der Feldgleichungen der Gravitation. Sitzungsberichte der Königlich Preussischen Akademie der Wissenschaften Berlin; Part 1; 1916; pp. 688-696. The Prusssian Academy of Sciences, Berlin, Germany.

[23] Nobel Prize, A. Press Release The Royal Swedish Academy of Sciences; 1993.The Royal Swedish Academy of Sciences, Stockholm, Sweden.

[24] Castelvecchi, D.; Witze, W. Einstein's gravitational waves found at last. Nature News 2016. 
[25] Binney, J.; \& Tremaine, S. Galactic Dynamics; Princeton University Press: Princeton, NJ, USA, 1987.

[26] Corbelli, E.; Salucci, P. The extended rotation curve and the dark matter halo of M33. Mon. Not. R. Astron. Soc. 2000, 311, 441-447, doi:10.1046/j.1365-8711.2000.03075.x.

[27] Milgrom, M. A modification of the Newtonian dynamics as a possible alternative to the hidden mass hypothesis. Astrophys. J. 1983, 270, 365-370, doi:10.1086/161130.

[28] Mannheim, P.D. \& Kazanas, D. Exact vacuum solution to conformal Weyl gravity and galactic rotation curves Astrophys. J. 1989, 342, 635.

[29] Mannheim, P.D. Linear Potentials and Galactic Rotation Curves Astrophys. J. 1993, 149, 150.

[30] Mannheim, P.D. Are Galactic Rotation Curves Really Flat? Astrophys. J. 1997, 479, 659.

[31] Tuval, M.; Yahalom, A. Newton's Third Law in the Framework of Special Relativity. Eur. Phys. J. Plus 2014, 129, 240, doi:10.1140/epjp/i2014-14240-x.

[32] Tuval, M.; Yahalom, A. Momentum Conservation in a Relativistic Engine. Eur. Phys. J. Plus 2016, 131, 374, doi:10.1140/epjp/i2016-16374-1.

[33] Yahalom, A. Retardation in Special Relativity and the Design of a Relativistic Motor. Acta Phys. Pol. A 2017, 131, 1285-1288.

[34] Asher Yahalom "Lorentz Symmetry Group, Retardation, Intergalactic Mass Depletion and Mechanisms Leading to Galactic Rotation Curves" Symmetry 2020, 12(10), 1693.

[35] Dodelson, Scott (2008). Modern cosmology (4 ed.). San Diego, CA: Academic Press. ISBN 978-0122191411. 